\title{
Escaping from nonhyperbolic chaotic attractors
}

\author{
Suso Kraut ${ }^{1}$ and Celso Grebogi ${ }^{1,2}$ \\ ${ }^{1}$ Instituto de Física, Universidade de São Paulo, Caixa Postal 66318, 05315-970 São Paulo, Brazil \\ ${ }^{2}$ Max-Planck-Institut für Physik komplexer Systeme, Nöthnitzer Strasse 38, 01187 Dresden, Germany
}

\begin{abstract}
The noise-induced escape process from a nonhyperbolic chaotic attractor is of physical and fundamental importance. We address this problem by uncovering the general mechanism of escape in the relevant low noise limit using the Hamiltonian theory of large fluctuations and by establishing the crucial role of the primary homoclinic tangency closest to the basin boundary in the dynamical process. In order to demonstrate that, we provide an unambiguous solution of the variational equations from the Hamiltonian theory. Our results are substantiated with the help of physical and dynamical paradigms, such as the Hénon and the Ikeda maps. It is further pointed out that our findings should be valid for driven flow systems and for experimental data.
\end{abstract}

PACS numbers: 05.45.Gg, 02.50.-r, 05.20.-y, 05.40.-a

Many nonequilibrium systems in physics, chemistry, biology or technology exhibit, as a crucial feature, noiseinduced escape from a metastable state. Examples range from Josephson junctions [1], switching in lasers [2], Penning traps 3], over chemical reactions [4] and protein folding [5] to electronic circuits [6, 7]. For small noise intensities, a WKB-like extension of Kramers' equilibrium theory [8, 9] has been developed to treat the realm of nonequilibrium systems [10, 11]. This approach, making use of an auxiliary Hamiltonian system, identifies the so-called most probable exit path (MPEP), whose probability of occurrence is exponentially larger than that of all the other paths. The MPEP can be observed through its 'prehistory probability distribution' and it was carried out numerically [13] and experimentially 7].

The variational equations for the calculation of the MPEP are well known both for continous 11, 12 and discrete systems [14, 15, 16, 17], yet it is in general not clear how to solve them. Methods have been proposed only for the special case of escaping from periodic states (fixed points, periodic orbits or limit cycles) [18, 19]. Thus the challenging question of noise-induced escape from a chaotic attractor (CA), although tried before, remained to be answered. The reason is that all previous work dealing with the escape from a CA relies on Monte Carlo simulations of the escape path. By making use of the 'prehistory probability distribution', an unstable periodic orbit embedded in the $\mathrm{CA}$ was identified and taken as initial condition for the calculation of the MPEP 20, 21, 22].

In this Letter we show that, in fact, the initial condition for the MPEP, and thus the path itself, is uniquely determined by the primary homoclinic tangency (PHT) closest to the basin boundary, as well as its preimages and images. Our solution of this long-standing problem does not only uncover the general mechanism of noiseinduced escape from a nonhyperbolic CA, but it reme- dies, in addition, shortcomings in the previous method. Herewith, we establish the following. First, no unstable periodic orbits have to be determined and no Monte Carlo simulations have to be run to identify these orbits in the escape path. Second, the arbitrariness of the selection of one particular periodic orbit, whose coincidence with the MPEP is not given from first principles, can be dispensed with. As a matter of fact, the PHT is, in contradistinction, a completetly deterministic quantity and can be both easily calculated numerically 23$]$ and extracted from experiments [24]. Third, complementary to the work of Ref. 22] in which the importance of the homoclinic structure of the fractal basin boundary to the MPEP is stressed, the present work closes an important gap in pointing out the essential role of the homoclinic structure of the chaotic attractor.

Virtually all CAs occurring in nature or serving as prototype models in nonlinear dynamics are nonhyperbolic. Nonhyperbolicity means that the stable and unstable manifolds of the system are tangent in the phase space. If both manifolds belong to the same periodic orbit, these tangencies are homoclinic. They are called primary, if the, generally quadratic, curvature of the manifolds in the vicinity of the tangent points attain a minimum. Bounded noise on nonhyperbolic CAs causes attractor deformations 23, 24], which are most pronounced at the forward and backward iterations of the PHT closest to the basin boundary. As a consequence, PHTs can be better defined as being those tangencies which exhibit an amplification of a pertubation under forward and backward dynamics.

In the following we consider discrete systems, but our results are not limited to them and apply equally well to any flow that can be reduced to a Poincaré map. For the nonequilibrium case, in analogy to Kramers' law, the mean first exit time is given through the least action $S$ [1, 17, 25] by $\langle\tau\rangle \sim \exp \left[\frac{S}{D}\right]$, where $D$ is the variance of 
the additive Gaussian white noise. To be specific, for a d-dimensional map $\mathbf{x}_{\mathbf{n}+\mathbf{1}}=\mathbf{f}\left(\mathbf{x}_{\mathbf{n}}\right)+\xi_{\mathbf{n}}$ the action of the escape trajectory to be minimized has the form

$$
S_{N}=\frac{1}{2} \sum_{n=1}^{N}\left[\mathbf{x}_{\mathbf{n}+\mathbf{1}}-\mathbf{f}\left(\mathbf{x}_{\mathbf{n}}\right)\right]^{2}=\frac{1}{2} \sum_{n=1}^{N} \xi_{\mathbf{n}}^{\mathbf{T}} \xi_{\mathbf{n}},
$$

where the $\xi_{\mathbf{n}}$ are the noise vector terms. The boundary conditions are such that $\mathbf{x}_{\mathbf{1}}$ is a point of the attractor and $\mathbf{x}_{\mathbf{N}}$ is on the basin boundary, from where no fluctuations are needed to pass to another stable state. The MPEP, which minimizes this action, can be calculated through the Lagrangian 12, 16]

$$
L=\frac{1}{2} \sum_{n=1}^{N} \xi_{\mathbf{n}}^{\mathbf{T}} \xi_{\mathbf{n}}+\sum_{n=1}^{N} \lambda_{\mathbf{n}}^{\mathbf{T}}\left(\mathbf{x}_{\mathbf{n}+\mathbf{1}}-\mathbf{f}\left(\mathbf{x}_{\mathbf{n}}\right)-\xi_{\mathbf{n}}\right)
$$

to yield upon variation of $\xi_{\mathbf{n}}, \lambda_{\mathbf{n}}$, and $\mathbf{x}_{\mathbf{n}}$ the areapreserving equations

$$
\begin{aligned}
\mathbf{x}_{\mathbf{n}+\mathbf{1}} & =\mathbf{f}\left(\mathbf{x}_{\mathbf{n}}\right)+\lambda_{\mathbf{n}} \\
\lambda_{\mathbf{n}+\mathbf{1}} & =\left\{\left(\mathbf{D f}\left(\mathbf{x}_{\mathbf{n}+\mathbf{1}}\right)\right)^{\mathbf{T}}\right\}^{-1} \lambda_{\mathbf{n}},
\end{aligned}
$$

where $\mathbf{D} \mathbf{f}$ is the Jacobian matrix of $\mathbf{f}$. The Lagrange multipliers $\lambda_{\mathbf{n}}$ replace the noise terms $\xi_{\mathbf{n}}$. The optimal solution of Eqs. (314) yields the least action $S=$ $\frac{1}{2} \sum_{n=1}^{N} \lambda_{\mathbf{n}}^{\mathbf{T}} \lambda_{\mathbf{n}}$, the corresponding MPEP (given by the $\left.\mathbf{x}_{\mathbf{n}}\right)$ and the optimal force $\left(\lambda_{\mathbf{n}}\right)$.

The solution of these equations is intricate though, caused by wild, fractal fluctuations of the energy landscape having many local minima and maxima 25]. A way to deal with these difficulties, when escaping from a periodic solution, is to employ a refined shooting method 18, 19]. It consists of a parameterization of the initial conditions $\mathrm{x}_{\mathbf{1}}$ in Eq. (3) on a small circle centered in one of the components of the periodic orbit $\mathbf{x}_{P O}$, and for $\lambda_{\mathbf{1}}$ pointing to the unstable manifold of $\mathbf{x}_{P O}$. Equivalently, one can take as an initial condition one point of the periodic orbit itself $\mathbf{x}_{\mathbf{1}}=\mathbf{x}_{P O}$ and $\lambda_{\mathbf{1}} \in r \times \phi$, where $\phi \in[0,2 \pi]$ and $r$ is varied within $r \in\left[r_{\text {min }}, L r_{\text {min }}\right]$. Here $L$ is the largest eigenvalue at $x_{P O}$ and $r_{\text {min }}$ arbitrary, but small. The upper limit of $r$ guarantees that every solution is only considered once. This provides an efficient numerical method for the calculation of the MPEP.

Now, we show that the same procedure can be applied to calculate the escape from a chaotic attractor. To do this, one has to select the initial condition $\mathbf{x}_{\mathbf{1}}$ as a preimage of the PHT closest to the basin boundary, since there noise causes the largest deviation 23]. This means that the energy to leave the CA is the lowest. The parameterization for $\lambda_{\mathbf{1}}$ remains unchanged, since every point on the CA has a well defined largest eigenvalue. We first demonstrate this for one of the funamental dynamical paradigms, the Hénon map [26]

$$
\begin{aligned}
x_{n+1} & =a-x_{n}^{2}+b y_{n}+\xi_{n} \\
y_{n+1} & =x_{n} .
\end{aligned}
$$

We choose the parameters $a=1.3$ and $b=0.29$ for which a $\mathrm{CA}$ and another attracting state at infinity coexist. In Fig. पthe CA is plotted, as well as its basin of attraction, and the saddle point on the boundary (square). We also include the PHT closest to the boundary together with its 10-fold preimages (circles) and the MPEP (crosses). The latter is found by iterating Eq. (3) at the 10-fold preimage of the PHT and by looking for the absolute minimum when changing $\lambda_{\mathbf{1}}$ as described above. As it can be seen, the MPEP moves initially very close to the deterministic dynamics yet it deviates increasingly with every iteration (as expected, since otherwise there were no escape). At the PHT the MPEP already differs con-

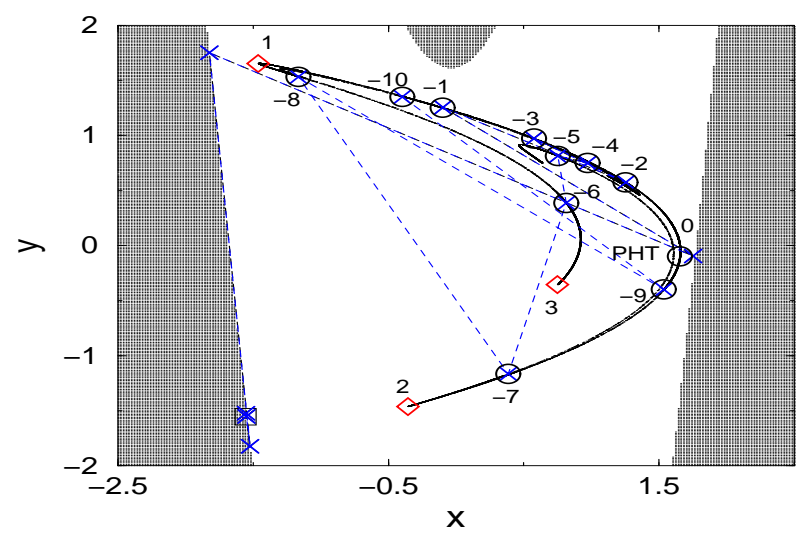

FIG. 1: (color online). CA for the Hénon map with $a=1.3$ and $b=0.29$. The hatched region markes the basin at infinity. The PHT at $(x, y)=(1.653,-0.099)$ is shown, together with its 10 -fold preimages (numbered $-1, . .,-10$ ) as circles, the 3 -fold images of the PHT (numbered 1,2,3) as diamonds, while the MPEP is depicted with crosses, connected through a dashed line to guide the eyes. The saddle point on the boundary is marked with a square.

siderably from it. Only there, it is for the first time located outside the CA and advances then, following the elongated images of the PHT (depicted as diamonds), to the basin boundary. From there it approaches the saddle point located on the basin boundary. We emphasize that it is not presupposed that the MPEP leaves the CA in the vicinity of the PHT. We simply use a preimage of the PHT as the initial condition $\mathbf{x}_{\mathbf{1}}$ and look for the optimal solution of Eqs. (34). The fact that the MPEP is indeed following very closely the structure of the PHT (preimages and images) is thus a confirmation of our claim.

To shed further light on this general mechanism, the stepwise action $S_{n}=\frac{1}{2} \lambda_{\mathbf{n}}^{\mathbf{T}} \lambda_{\mathbf{n}}$ is plotted in Fig. 2) There 


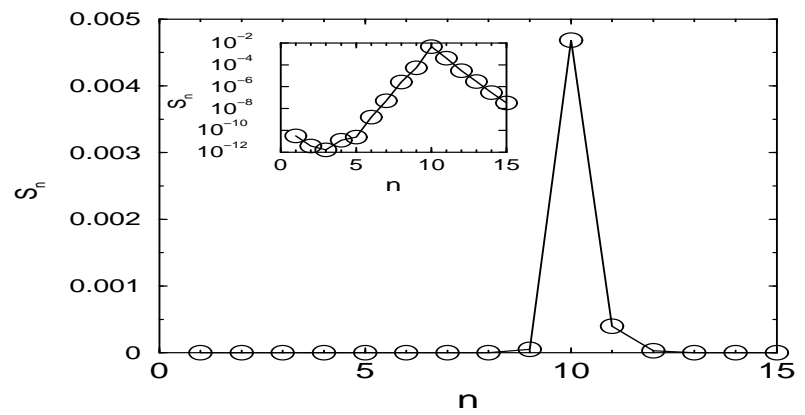

FIG. 2: The stepwise action $S_{n}=\frac{1}{2} \lambda_{\mathbf{n}}^{\mathbf{T}} \lambda_{\mathbf{n}}$ for the Hénon map. At each time step, the value of the graph corresponds to the optimal force $\lambda_{\mathbf{n}}$, as a result of the optimal solution of Eqs. (34). The inset shows the data in semi-log scale.

is only one large peak (step 10) where the MPEP moves away from the CA close to the PHT. Afterwards, the required energy drops off exponentially, since the MPEP follows a relaxational path. It is important to note that this scenario differs from the one of the attractor deformation. In the latter, the trajectory deviates from the CA starting at the PHT, while in our case the MPEP departs already at the preimages (cf. Fig. 2] inset) and is, when passing the PHT, a finite distance away. This is the reason why we have to start iterating Eq. (3) at some preimage of the PHT. However, also by using only the 5 -fold preimage of the PHT the same MPEP was obtained. Thus neither the exact number of preimages nor the accurate determination of the PHT is crucial - an indication about the robustness of the method.

In Fig. [3 the MPEP is compared with a direct Monte Carlo simulation of the escape trajectory. The simulation was carried out with a noise strength of $D=2.4 \times 10^{-4}$, resulting in a trajectory of length $2.5 \times 10^{12}$. One sees a strikingly good agreement of about 15 iterations before the path approaches the saddle point, reaching back to the 10-th preimage of the PHT. This remarkable coincidence of the simulated path with the MPEP corroborates the general validity of the procedure.

To connect the MPEP with the low-period unstable periodic orbits embedded in the $\mathrm{CA}$, we calculate all periodic orbits up to period 20 27]. Only one periodic orbit is found to be close to the MPEP, having period 9. It is also included in Fig. 3. Thus, if one had adopted the previous method of finding periodic orbits close to the MPEP by using stochastic simulations of the system, one might have been led to conclude that the MPEP starts at that period 9 orbit. However, such a reasoning obscures the general deterministic structure of the MPEP with respect to the PHT, as presented in this work.

Next we exemplify the method with the Ikeda map [28], a discrete model of a laser pulse in an optical cavity.

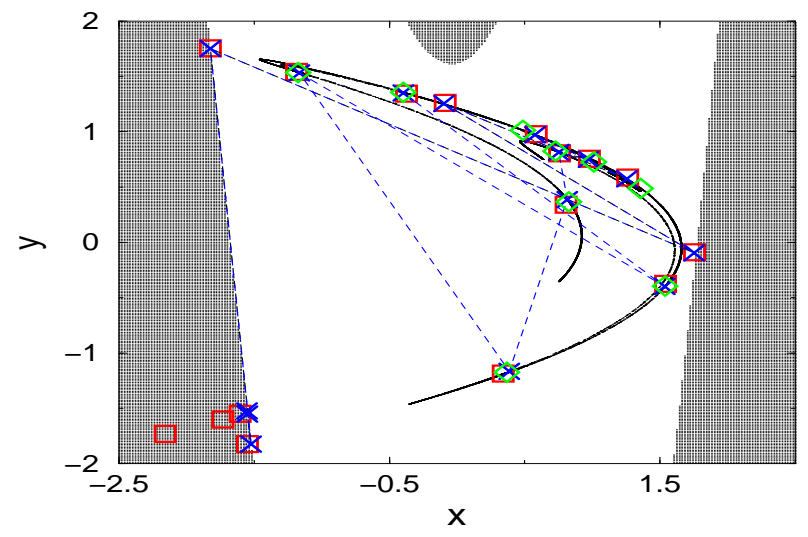

FIG. 3: (color online). The MPEP is depicted with crosses, connected through a dashed line to guide the eyes. The escape path, as obtained from a Monte Carlo simulation with a noise strength of $D=2.4 \times 10^{-4}$, is shown with squares. An unstable period- 9 orbit is displayed with diamonds.

With complex variables it has the form

$$
\mathbf{z}_{\mathbf{n}+\mathbf{1}}=a+b \mathbf{z}_{\mathbf{n}} \exp \left[i \kappa-\frac{i \eta}{1+\left|\mathbf{z}_{\mathbf{n}}\right|^{2}}\right]+\xi_{\mathbf{n}},
$$

where $\mathbf{z}_{\mathbf{n}}=x_{n}+i y_{n}$ is related to the amplitude and phase of the $n t h$ laser pulse exiting the cavity. The parameter $a$ is the laser input amplitude and $(1-b)$ the damping, while the empty cavity detuning is given by $\kappa$ and the detuning due to a nonlinear dielectric medium by $\eta$.

We fix the parameters at $a=0.92, b=0.9, \kappa=0.4$ and $\eta=6.0$. For the noisefree system, two stable states are present, a fixed point and a CA. In Fig. 4 we present the result for the Ikeda map, analogously to Fig. [1 The $\mathrm{CA}$, the basin of attraction and the saddle point on the boundary (square) are depicted. It is also shown the PHT closest to the boundary together with its 5 -fold preimages (circles) and the MPEP (crosses). We include two period-5 orbits as well (triangles), which are located near the images of the PHT. The behaviour of the MPEP and the optimal force (not shown) is qualitatively the same up to where the path reaches the PHT. Then it passes very close to two unstable period- 5 orbits, which are outside the $\mathrm{CA}$, before it approaches the basin boundary. This is so because they happen to be located exactly at the regions of phase space where the noise elongations attain a maximum, which occurs close to the images of the PHT 23]. The passing of the MPEP through another invariant set was also found in a different system [21], as well in the more complex situation when there exists a chaotic saddle 29]. It does not happen for the Hénon map though, since all the unstable periodic orbits lie, for the present parameter values, on the CA.

To conclude, we have unveiled the general mechanism 


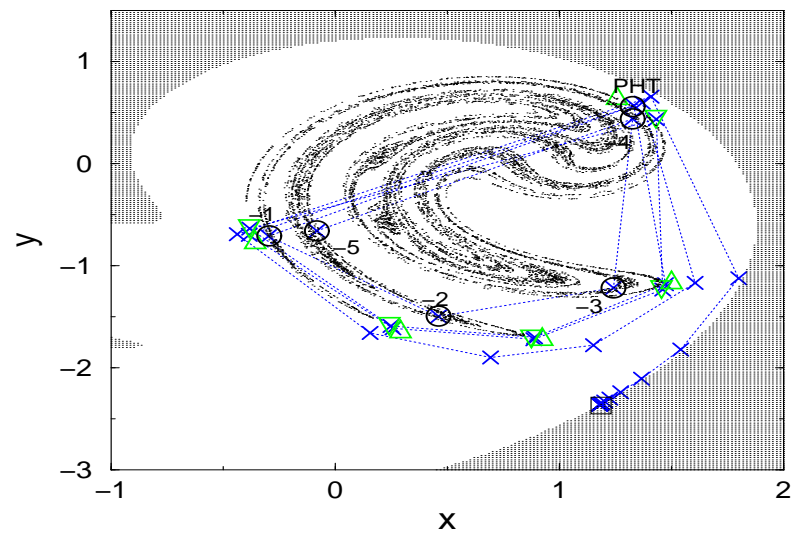

FIG. 4: (color online). CA for the Ikeda map with $a=$ $0.92, b=0.9, \kappa=0.4$, and $\eta=6.0$. The hatched region marks the basin of the fixed point located at $(x, y)=(2.995,3.947)$. Circles depict the PHT at $(x, y)=(1.327,0.559)$ together with its 5 -fold preimages (numbered $-1, . .,-5$ ). The MPEP is shown with crosses, connected through a dashed line to guide the eyes. In addition, two unstable period- 5 orbits are displayed with upward and downward triangles, respectively. The saddle point on the boundary is marked with a square.

of noise-induced escape from a nonhyperbolic CA. It is shown that the MPEP exits at the vicinity of the PHT closest to the basin boundary. Our findings are established by solving unambiguously the auxiliary Hamiltonian system, which yields the exact description in the low noise limit. This has, for the first time, been established without taking recourse to Monte Carlo simulations, and only the knowledge of the deterministic structure of the $\mathrm{PHT}$ is required. This mechanism gives a robust practical procedure and should also be able to be confirmed experimentally [7, 24]. It is advantageous both for stabilizing systems and for energy-optimal switching between different states (cf. [21]).

We acknowledge D. G. Luchinsky, S. Beri, U. Feudel, M. S. Baptista, and H. Kantz for valuable discussions. This work was supported by the Alexander von Humboldt Stiftung.

[1] M. H. Devoret, D. Esteve, J. M. Martinis, A. Cleland, and J. Clarke, Phys. Rev. B 36, 58 (1977).
[2] J. Hales, A. Zhukov, R. Roy, and M. I. Dykman, Phys. Rev. Lett. 85, 78 (2000).

[3] L. J. Lapidus, D. Enzer, and G. Gabrielse, Phys. Rev. Lett. 83, 899 (1999).

[4] D. T. Gillespie, J. Chem. Phys. 81, 2340 (1977).

[5] A. Sali, E. Shakhnovich, and M. Karplus, Nature (London) 369, 248 (1994).

[6] R. N. Mantegna and B. Spagnolo, Phys. Rev. Lett. 76, 563 (1996).

[7] D. G. Luchinsky and P. V. E. McClintock, Nature (London) 389, 463 (1997).

[8] H. A. Kramers, Physica (Utrecht) 7, 284 (1940).

[9] P. Hänggi, P. Talkner, and M. Borkovec, Rev. Mod. Phys. 62, 251 (1990); V. I. Mel'nikov, Phys. Rep. 209, 1 (1991).

[10] L. Onsager and S. Machlup, Phys. Rev. 91, 1505 (1953).

[11] M. I. Freidlin and A. D. Wentzell, Random perturbations of dynamical systems, Springer Verlag, Berlin, 1984.

[12] M. I. Dykman, Phys. Rev. A 42, 2020 (1990).

[13] M. I. Dykman, P. V. E. McClintock, V. N. Smelyanskiy, N. D. Stein, and N. G. Stocks , Phys. Rev. Lett. 68, 2718 (1992).

[14] R. L. Kautz, Phys. Lett. A 125, 315 (1987).

[15] P. D. Beale, Phys. Rev. A 40, 3998 (1989).

[16] P. Grassberger, J. Phys. A 22, 3283 (1989).

[17] R. Graham, A. Hamm, and T. Tél, Phys. Rev. Lett. 66, 3089 (1991).

[18] D. G. Luchinsky, S. Beri, R. Mannella, P. V. E. McClintock, and I. A. Khovanov Int. J. Bifurcation Chaos Chaos Appl. Sci. Eng. 12, 583 (2002).

[19] S. Beri, D. G. Luchinsky, R. Mannella, and P. V. E. McClintock, submitted to Phys. Rev. E .

[20] D. G. Luchinsky and I. A. Khovanov, JETP Lett. 69, 825 (1999).

[21] I. A. Khovanov, D. G. Luchinsky, R. Mannella, and P. V. E. McClintock, Phys. Rev. Lett. 85, 2100 (2000).

[22] A. N. Silchenko, S. Beri, D. G. Luchinsky, and P. V. E. McClintock, Phys. Rev. Lett. 91, 174104 (2003).

[23] L. Jaeger and H. Kantz, Physica D 105, 79 (1997).

[24] M. Diestelhorst, R. Hegger, L. Jaeger, H. Kantz, and R.P. Kapsch, Phys. Rev. Lett. 82, 2274 (1999).

[25] R. Graham and T. Tél, Phys. Rev. Lett. 52, 9 (1984).

[26] M. Hénon, Comm. Math. Phys. 50, 69 (1976).

[27] O. Biham and W. Wenzel, Phys. Rev. Lett. 63, 819 (1989).

[28] K. Ikeda, Opt. Commun. 30, 257 (1979); S. M. Hammel, C. K. R. T. Jones, and J. V. Maloney, J. Opt. Soc. Am. B 2, 552 (1985).

[29] S. Kraut and U. Feudel, Phy. Rev. E 66, 015207(R) (2003); Physica D 181, 222 (2003). 